\title{
Modelo para estimar a penetração de cloretos em concreto com perfil formando pico: verificação da sua precisão
}

\author{
GUIMARÃES ${ }^{\mathrm{I}}$,A.T.C.; HELENE ${ }^{\mathrm{II}}$, P.R.L. \\ ${ }^{\text {I }}$ Escola de Engenharia - FURG - Rua João Manoel 38, apto 701 CP: 96211-060, Rio Grande, RS. \\ e-mail: atcg@vetorial.net \\ II Departamento de Construção Civil - PCC-USP - Av. Prof. Almeida Prado - Trav. 2 - Cidade \\ Universitária, CP 05508-900, São Paulo, SP \\ e-mail: paulo.helene@pcc.usp.br
}

\section{RESUMO}

Ataque de cloretos em ambiente marítimo ou em estruturas que são usados sais de degelo nem sempre apresentam perfis com teores diminuindo da superfície externa para o interior do concreto. Alguns perfis apresentam um aumento no teor de cloretos da superfície externa até uma determinada profundidade, diminuindo deste ponto até camadas mais profundas, formando pico. Este tipo de perfil deve ter tratamento diferente do modelo tradicional da segunda lei de Fick, obtendo modelos de estimativa de vida útil mais precisos.

Modelo previamente proposto por GUIMARÃES e HELENE estima a penetração de íons cloreto ao longo do tempo para perfis formando pico. Para comprovar a eficiência deste modelo é necessário observar o comportamento do perfil de cloretos com pico de uma mesma estrutura ao longo do tempo. Com este objetivo foram obtidos dois perfis de cloretos de uma mesma estrutura em idades diferentes, 22 anos e 27 anos. Sobre o perfil obtido na idade de 22 anos foram estimados perfis para a idade de 27 anos através de três modelos: a)modelo tradicional considerando a segunda lei de Fick, extrapolando o valor de $\mathrm{C}_{\mathrm{S}}$; b)modelo tradicional considerando a segunda lei de Fick, deslocando o eixo do x para a profundidade do pico e c) modelo proposto por GUIMARÃES e HELENE. Os resultados são comparados com o perfil medido na idade de 27 anos, sendo os resultados analisados.

O modelo apresentou uma boa precisão, sendo que deve ser aferido em outras estruturas em uso.

Palavras-chaves: concreto, cloreto, perfil, pico.

\section{Model to predict the chloride penetration in concrete with profile forming peak: verification of its precision}

\section{ABSTRACT}

Chloride attack in marine environments or in structures where deicing salts are used will not always show profiles with concentrations that decrease from the external surface to the interior of the concrete. Some profiles show an increase in chloride concentrations from when a peak is formed. This type of profile must be analyzed in a different way from the traditional model of Fick's second law to generate more precise service life models.

A model for forecasting the penetration of chloride ions as a function of time for profiles having formed a peak. To confirm the efficiency of this model, it is necessary to observe the behavior of a chloride profile with peak in a specific structure over a period of time. To achieve this, two chloride profiles with different ages (22 and 27 years) were extracted from the same structure. The profile obtained from the 22year sample was used to estimate the chloride profile at 27 years using three models: a) the traditional model using Fick's second law and extrapolating the value of $C_{S}$ - external surface chloride concentration; b) the traditional model using Fick's second law and shifting the x-axis to the peak depth; c) the previously proposed model. The results from these models were compared with the actual profile measured in the 27year sample and the results were analyzed.

The model was presented with good precision for this study of case, requiring to be tested with other structures in use. 
Keywords: concrete, chloride, profile, peak.

\section{INTRODUÇÃO}

Ataque de cloretos em ambiente marítimo ou em estruturas que são usados sais de degelo nem sempre apresentam perfis com teores diminuindo da superfície externa para o interior do concreto. Alguns perfis apresentam um aumento no teor de cloretos da superfície externa até uma determinada profundidade, diminuindo deste ponto até camadas mais profundas, formando pico. Este tipo de perfil deve ter tratamento diferente do modelo tradicional da segunda lei de Fick, obtendo modelos de estimativa de vida útil mais precisos.

Conforme ANDRADE et al. [1]] e NILSSON et al. [2], o comportamento da penetração de cloretos após a formação do pico, continua com um comportamento similar a solução da segunda lei de Fick, considerando o valor do teor de cloretos no pico constante.

Modelo sugerido por NILSSON et al. [2] calcula por regressão um valor de Cs teórico para o perfil, considerando apenas os teores de cloretos de profundidades iguais ou maiores que a profundidade do pico (Fig. 1). Após definir este perfil estima-se a penetração de cloretos considerando este valor de Cs teórico. Este modelo estima uma aumento do valor da concentração de cloretos no pico para idades mais avançadas, enquanto modelo sugerido por ANDRADE et al. [1] considera que após uma idade o teor de cloretos no pico deve permanecer constante, como será descrito logo a seguir.

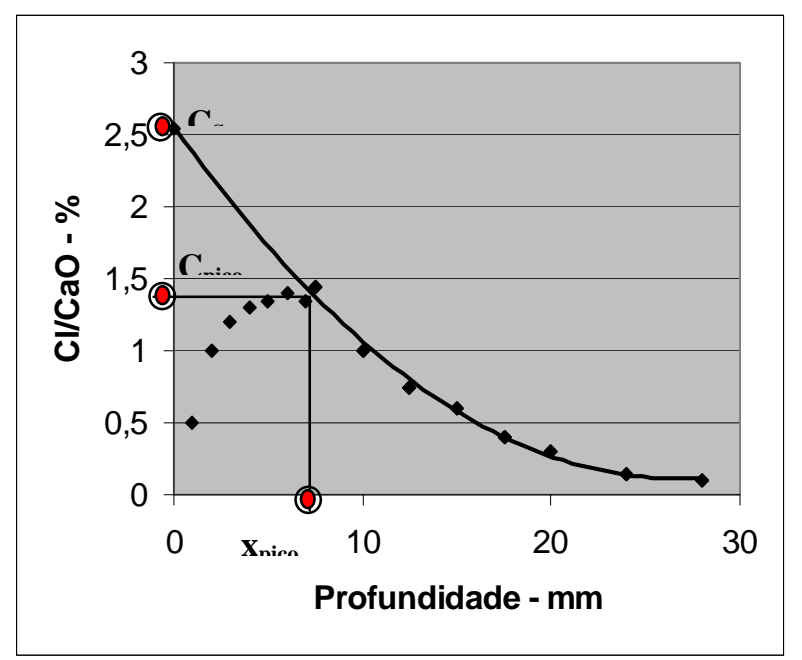

Figura 1: Perfil teórico quando o perfil real apresenta pico [2].

Para permitir a previsão do comportamento em idades mais avançadas mantendo o teor no pico constante e seguindo as leis de Fick, foi adaptado modelo apresentado por CRANK [5] para estimar a penetração de cloretos ao longo do tempo para perfis formando pico [ $\underline{3}$, 4], conforme será discutido mais adiante.

O objetivo deste trabalho é verificar a eficiência deste modelo para estimar a vida útil residual de estruturas de concreto armado ou calcular seu coeficiente de difusão, permitindo prever a vida útil de projeto de estruturas a serem executadas com concreto similar.

\section{MÉTODOS E MODELOS PARA ESTIMATIVA DE VIDA ÚTIL}

Para comprovar a eficiência do modelo de GUIMARÃES e HELENE [4] é necessário observar o comportamento do perfil de cloretos com pico de uma mesma estrutura ao longo do tempo. Para tal, foram obtidos dois perfis de cloretos de uma mesma estrutura em idades diferentes, 22 anos e 27 anos. Sobre o perfil obtido na idade de 22 anos foram estimados perfis para a idade de 27 anos através de três modelos: a)modelo tradicional considerando a segunda lei de Fick, extrapolando o valor de CS, conforme proposto por NILSSON et al. [2]; b)modelo tradicional considerando a segunda lei de Fick, deslocando o eixo do x para a profundidade do pico, conforme proposto por ANDRADE et al. [1] , e c) modelo proposto por GUIMARÃES e HELENE [4]. Os resultados são comparados com o perfil medido na idade de 27 anos, sendo os resultados analisados. 


\subsection{Modelo tradicional correspondente a Segunda Lei de Fick}

Modelo de ANDRADE et al. [1] considera que, para concreto com considerável tempo de ataque a cloretos, a concentração no pico deve ser constante e o ponto zero do eixo do $\mathrm{x}$ deve ser transferido para a profundidade onde forma o pico de teor de cloretos (Fig. 2).

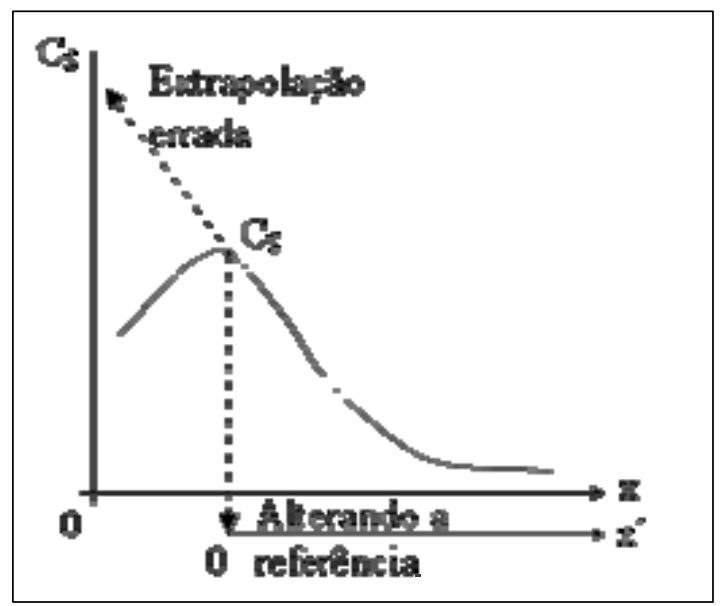

Figura 2: Correta e errônea extrapolação dos teores do perfil de cloretos [1]].

Este modelo [1] também considera que a curva a partir do pico até camadas mais profundas apresenta comportamento igual ao proposto pela solução tradicional da segunda lei de Fick.

Portanto pode-se utilizar a solução tradicional referente à segunda lei de Fick, conforme eq. 1, considerando $\mathrm{x}$ igual a zero para a profundidade do pico.

$$
\frac{C c_{C l}-C_{O}}{C_{\text {Seq }}-C_{O}}=\operatorname{erfc} \frac{x^{\prime}}{2 \sqrt{D \cdot t}}
$$

onde:

$C c_{C l}=$ teor de cloretos na posição x';

$C_{O}=$ teor inicial de cloretos na posição x';

$\mathrm{C}_{\mathrm{seq}}=$ teor de cloretos no pico $\left(\mathrm{x}^{\prime}=\right.$ zero);

$x^{\prime}$ = distância entre o pico e a posição do teor $\mathrm{Cc}_{\mathrm{Cl}}$, ou seja, considerando $\mathrm{x}^{\prime}=0$ no pico;

$\mathrm{D}=$ coeficiente de difusão de cloretos no concreto;

$t=$ tempo, considerando $\mathrm{t}=$ zero aquele instante em que o concreto começa a sofrer $\mathrm{o}$ ataque de cloretos, ou seja, o tempo de exposição da estrutura a ambiente marítimo ou a sais de degelo;

$\operatorname{erfc}(\mathrm{z})=1-\operatorname{erf}(\mathrm{z})$;

$\operatorname{erf}(\mathrm{z})$ = função erro de Gauss.

\subsection{Modelo proposto por Guimarães e Helene}

Modelo de GUIMARÃES e HELENE [3, 4 ] também considera que o ponto zero do eixo do x deve ser transferido para a profundidade onde forma o pico de teor de cloretos (Fig. 2) e que a partir deste ponto, em direção a camadas mais profundas, o perfil apresenta um comportamento conforme a segunda lei de Fick. Este modelo [3,4] consideram ainda que a forma de um pico sugere um efeito equivalente à difusão no sentido das maiores profundidades, a partir desse pico, com perda de parte do material que sofre difusão na direção oposta (Fig. 3). 


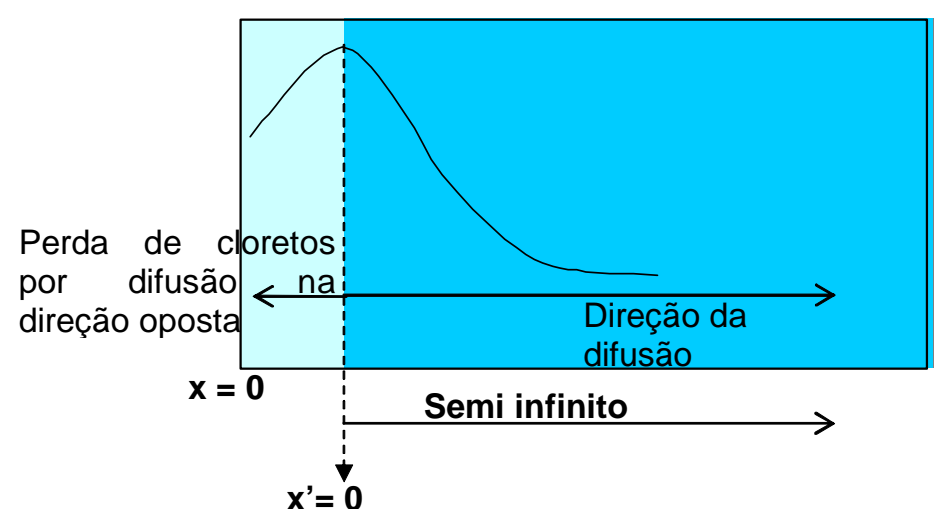

Figura 3: Difusão de cloretos com perda de fração de cloretos que sofre difusão na direção oposta []]

Modelo apresentado por GUIMARÃES e HELENE [4]], adaptado da equação de CRANK [్] apresenta a seguinte equação para essa condição de difusão:

$$
-D \frac{\partial C}{\partial x}=\alpha\left(C_{S e q}-C_{P}\right)
$$

onde:

$\mathrm{C}_{\mathrm{Seq}}=$ teor equivalente de cloretos na superfície externa do concreto;

$\mathrm{C}_{\mathrm{P}}=$ teor de cloretos no pico;

$\alpha=$ constante de proporcionalidade.

O modelo de GUIMARÃES e HELENE [4]], ainda baseado em CRANK [్], apresenta a seguinte solução para a eq. 2:

$$
\frac{C c_{C l}-C_{O}}{C_{\text {Seq }}-C_{O}}=\operatorname{erfc} \frac{x^{\prime}}{2 \sqrt{D \cdot t}}-\exp \left(h x^{\prime}+h^{2} D \cdot t\right) \cdot \operatorname{erfc}\left(\frac{x^{\prime}}{2 \sqrt{D \cdot t}}+h \sqrt{D \cdot t}\right)
$$

onde:

$\mathrm{Cc}_{\mathrm{Cl}}=$ teor de cloretos na posição x’;

$\mathrm{C}_{\mathrm{O}}=$ teor inicial de cloretos na posição x';

$\mathrm{x}^{\prime}$ = distância entre o pico e a posição do teor $\mathrm{Cc}_{\mathrm{Cl}}$, ou seja, considerando $\mathrm{x}^{\prime}=0$ no pico;

$\mathrm{h}=\alpha / \mathrm{D}$

$\mathrm{D}$ = coeficiente de difusão do concreto;

$\mathrm{t}$ = tempo, considerando $\mathrm{t}=$ zero aquele instante em que o concreto começa a sofrer $\mathrm{o}$ ataque de cloretos, ou seja, o tempo de exposição da estrutura a ambiente marítimo ou a sais de degelo;

$\operatorname{erfc}(z)=1-\operatorname{erf}(z)$;

$\operatorname{erf}(\mathrm{z})=$ função erro de Gauss.

Conforme demonstra CRANK [] é possível obter-se curvas de $\left.\left(\mathrm{Cc}_{\mathrm{Cl}}-\mathrm{C}_{\mathrm{O}}\right) / \mathrm{C}_{\mathrm{Seq}}-\mathrm{C}_{\mathrm{O}}\right)$ em relação a (x' / 2(D.t $)^{1 / 2}$ ), visto que para cada relação $C_{P} / C_{\text {Seq }}$ (considerando $C_{O}$ igual a zero) tem-se um valor característico de h(D.t) $)^{1 / 2}$ para a curva (Fig. 4). 


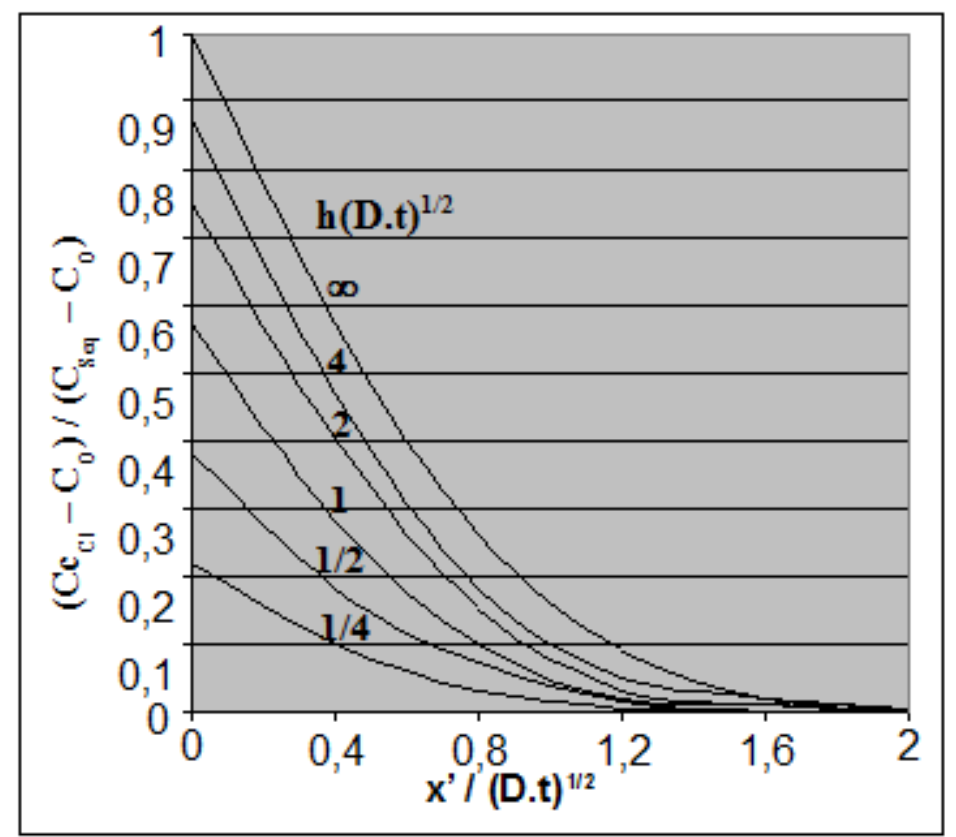

Figura 4: Curvas de relação entre $\left.\left(\mathrm{CC}_{\mathrm{Cl}}-\mathrm{C}_{\mathrm{O}}\right) / \mathrm{C}_{\mathrm{Seq}}-\mathrm{C}_{\mathrm{O}}\right)$ e $\left(\mathrm{x}^{\prime} / 2(\mathrm{D} . \mathrm{t})^{1 / 2}\right)[\underline{5}]$

Este modelo representa apenas o comportamento do perfil de cloretos somente do pico para o interior do concreto. Em relação ao modelo sugerido por ANDRADE et al. [1], o modelo sugerido por GUIMARÃES e HELENE [4] estima valores diferentes de coeficiente de difusão (D), sendo que este último considera a perda de cloretos no sentido contrário da difusão (Fig. 3), o que ocorre em certas situações, como o estudo de caso apresentado a seguir.

\section{APLICAÇÃO DOS MODELOS EM ESTRUTURA EXISTENTE}

\subsection{Estrutura pesquisada}

O elemento estrutural utilizado nesta pesquisa é um trecho composto de estacas pranchas do cais do Terminal de Conteiners-TECON. O cais está localizado em porto marítimo da cidade do Rio Grande - Brasil (Fig. 5), junto ao canal do Rio Grande. Este canal liga o estuário da Lagoa dos Patos, com aproximadamente 300 km de extensão, ao Oceano Atlântico.

Através das medições diárias durante o ano de 1992, do marégrafo localizado próximo à estrutura pesquisada e com sistema de canaletas própria para medir a máxima altura diária de respingo, BRETANHA [6] classificou o micro-ambiente dos pontos pesquisados. Nestes pontos, durante o ano, são predominantes os dias de zona de névoa (50\%) e zona de respingo (45\%) e poucos dias de zona de maré (5\%).

Durante os meses de verão a salinidade da água do canal é alta, atingindo $34 \%$, enquanto que no inverno a salinidade é muito próxima a zero na maioria dos dias. Devido ao regime de ventos e chuvas na região, o nível da água no canal é normalmente mais elevado no inverno, provocando respingos mais constantes e mais fortes. Este processo provoca ciclos de secagem e molhagem com água com baixíssima salinidade, que deve provocar a dessanilização na camada mais externa do concreto. Esta condição é propícia para formação de perfis de cloretos com pico. 


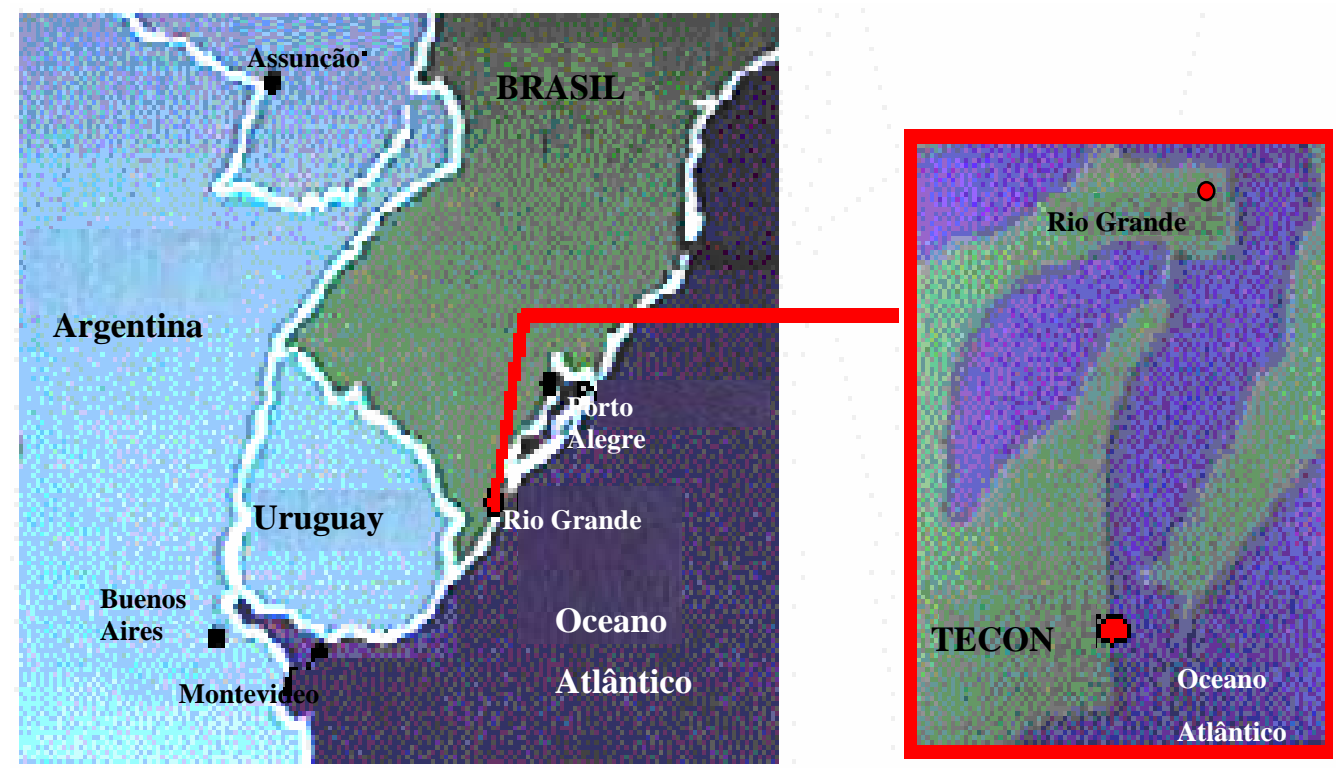

Figura 5: Localização do cais do Terminal de Conteiners - TECON, RGS, Brasil

\subsection{Características do concreto}

Testemunhos foram extraídos conforme ASTM C 42 [7], e realizados ensaios de caracterização, além dos dados obtidos em relatórios de execução da obra. Obteve-se o perfil do teor de cloretos com material extraído com furadeira a cada $5 \mathrm{~mm}$ de profundidade (20 furos em seis estacas, totalizando 120 furos, de um trecho de $50 \mathrm{~m}$ do cais).

O concreto da estrutura foi executado com agregado graúdo de origem granítica britada com diâmetro máximo $\left(\mathrm{D}_{\text {máx }}\right)$ de $38 \mathrm{~mm}$ e a areia é quartzosa. O aglomerante utilizado foi CP IV-25 com 34\% de cinza volante com um consumo de $400 \mathrm{~kg} / \mathrm{m}^{3}$. A relação a/c é de 0,42 obtendo-se um $\mathrm{f}_{\mathrm{ck}}$ de $19 \mathrm{MPa}$. Com 22 anos de uso apresentou massa específica de $2190 \mathrm{~kg} / \mathrm{m}^{3}$ e absorção por imersão e após fervura de 7,09 \%, sendo os ensaios executados conforme ASTM C 642 [8]. O cobrimento da grande maioria das barras de aço é igual ou superior a $60 \mathrm{~mm}$.

Embora GUIMARÃES [9] já tivesse obtido o perfil de cloretos na idade de 22 anos da estrutura pesquisada, esses teores foram refeitos com amostras pulverizadas devidamente armazenadas, juntamente com as amostras extraídas na idade de 27 anos. Esse procedimento foi adotado para que os perfis comparados fossem ensaiados nas mesmas condições, ou seja, no mesmo laboratório e executado pelo mesmo laboratorista. Os teores de cloretos solúveis em água em relação à massa de concreto, obtidos conforme ASTM 1218 [10], em amostras extraídas aos 22 anos de idade, foram de 0,28\%, 0,42\%, 0,57\%, 0,44\%, $0,32 \%, 0,19 \%, 0,11 \%, 0,07 \%, 0,04 \%$ e $0,01 \%$, respectivamente para as profundidades de $2,5 \mathrm{~mm}, 7,5 \mathrm{~mm}$, $12,5 \mathrm{~mm}, 17,5 \mathrm{~mm}, 22,5 \mathrm{~mm}, 27,5 \mathrm{~mm}, 32,5 \mathrm{~mm}, 37,5 \mathrm{~mm}, 42,5 \mathrm{~mm}$ e $47,5 \mathrm{~mm}$. Para a idade de 27 anos os teores para as mesmas profundidades foram respectivamente de $0,41 \%, 0,54 \%, 0,60 \%, 0,45 \%, 0,35 \%$, $0,28 \%, 0,15 \%, 0,08 \%, 0,07 \%$ e $0,06 \%$. O teor de $0,07 \%$ de cloretos em relação à massa de concreto equivale a $0,4 \%$ de cloretos em relação à massa de cimento para o traço do concreto pesquisado.

\subsection{Aplicação dos modelos}

Foram comparadas aplicações dos três modelos descritos:

- equação tradicional da solução da segunda lei de Fick, utilizando Cs extrapolado para a superfície da estrutura, sugerido por NILSSON et al. [2], conforme Figura 1, utilizando a eq. 1;

- equação tradicional da solução da segunda lei de Fick, considerando x` igual a zero no pico, sugerido por ANDRADE et al. [1] , conforme Figura 2, utilizando a eq. 1;

- proposta de GUIMARÃES e HELENE [4], utilizando eq. 3.

Utilizando o método do mínimo quadrado foi realizada a regressão dos valores do perfil medido na idade de 22 anos utilizando os três métodos, obtendo-se três curvas com valores característicos da Tabela 1. 
A Fig. 6 apresenta valores dos perfis medidos nas idades de 22 anos e 27 anos; curva de regressão obtida pelo modelo tradicional deslocando o eixo do x para o pico para idade de 22 anos; perfis estimados para 27 anos e 100 anos com modelo tradicional deslocando o eixo do x para o pico, conforme sugerido por ANDRADE et al. [1] ; curva de regressão obtida pelo modelo tradicional extrapolando o valor de Cs para idade de 22 anos; e perfis estimados para 27 anos e 100 anos com modelo tradicional extrapolando o valor de Cs, conforme sugerido por NILSSON et al. [2] ], respectivamente às curvas citadas na legenda.

Tabela 1: Valores obtidos por regressão para os três modelos analisados na idade de 22 anos

\begin{tabular}{|c|c|c|c|}
\hline Modelos & $\begin{array}{l}D_{2} \\
\left(\mathrm{~mm}^{2} \text {.ano }^{-1}\right)\end{array}$ & $\begin{array}{l}C_{S} \\
(\%)\end{array}$ & $\begin{array}{l}C_{\text {pico }} \\
(\%)\end{array}$ \\
\hline $\begin{array}{l}\text { Tradicional - Cs extrapolado } \\
-\quad \text { eq. } 1 \text { - sugerido por } \\
\text { NILSSON et al. [2] }\end{array}$ & 9,53 & 1,09 & 0,59 \\
\hline $\begin{array}{l}\text { Tradicional }-\mathrm{x} \text { extrapolado - } \\
\text { eq. } 1-\text { sugerido por } \\
\text { ANDRADE } \text { et } \text { al. }[\underline{1}]\end{array}$ & 5,74 & $0,577 *$ & $0,577 *$ \\
\hline $\begin{array}{l}\text { Proposta de Guimarães et al. } \\
\text { [4] - eq. } 03\end{array}$ & 7,07 & 0,785 & 0,582 \\
\hline
\end{tabular}

*CS = Cpico, pois considera x’ igual a zero, ou seja os dois valores se referem a posição do pico

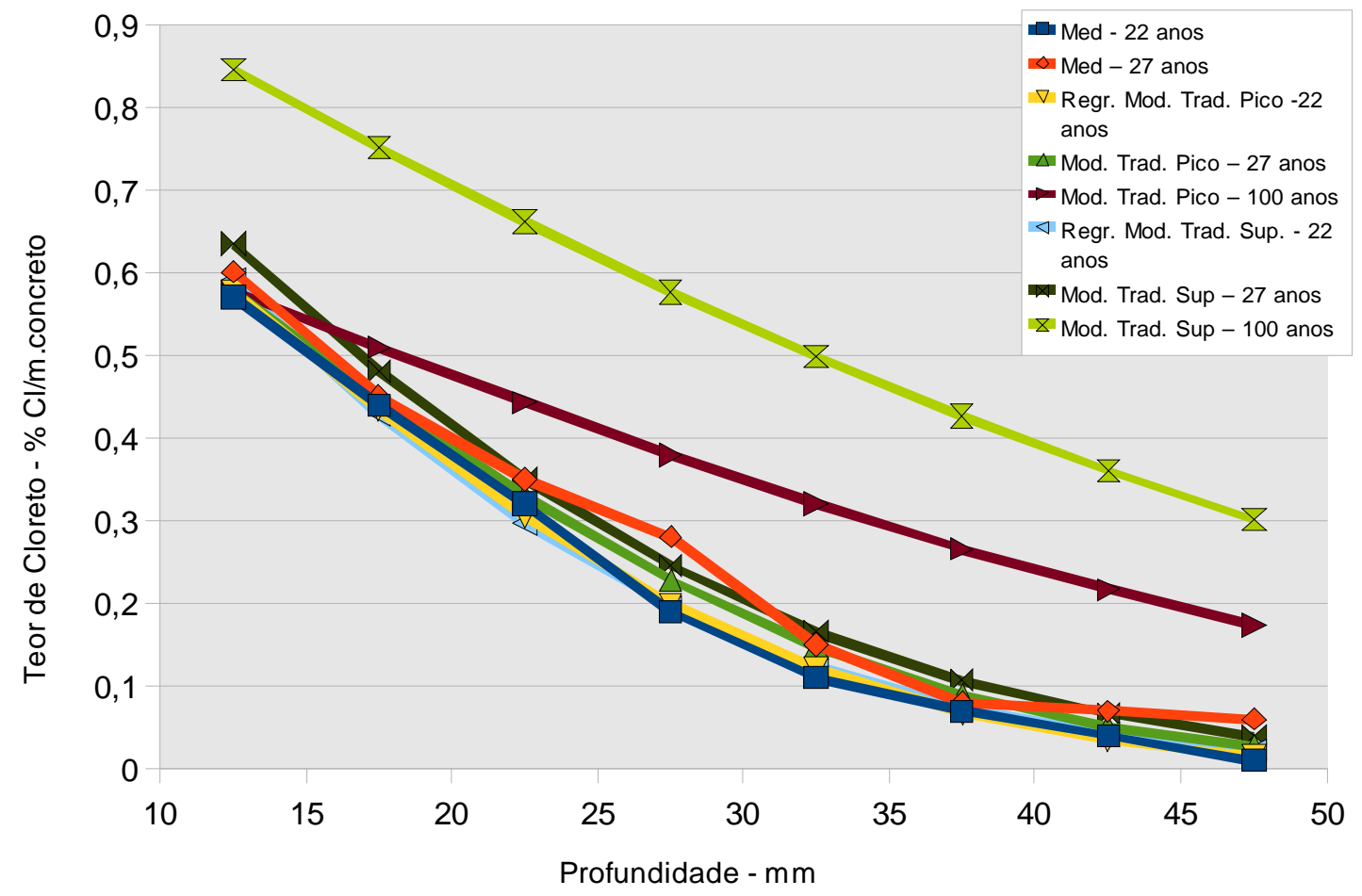

Figura 6: Perfis de cloretos medidos nas idades de 22 anos e 27 anos; curva de regressão obtida pelo modelo tradicional deslocando o eixo do x para o pico para idade de 22 anos; perfis estimados para 27 anos e 100 anos com modelo tradicional deslocando o eixo do x para o pico, conforme sugerido por

ANDRADE et al. [1], curva de regressão obtida pelo modelo tradicional extrapolando o valor de Cs para idade de 22 anos; e perfis estimados para 27 anos e 100 anos com modelo tradicional extrapolando o valor de Cs, conforme sugerido por NILSSON et al. [2]], respectivamente às curvas citadas na legenda. 
Na Fig. 6 observa-se que para o modelo tradicional posicionando o eixo do x no pico mantém-se constante o valor do teor de cloretos neste ponto.

A Fig. 7 apresenta valores medidos nas idades de 22 anos e 27 anos; curva de regressão obtida pelo modelo tradicional deslocando o eixo do x para o pico para idade de 22 anos; perfis estimados para 27 anos e 100 anos com modelo tradicional deslocando o eixo do x para o pico, conforme sugerido por ANDRADE et al. [1]], perfis estimados para 27 anos e 100 anos utilizando modelo sugerido por GUIMARÃES e HELENE [4], e curva de regressão obtida pelo modelo tradicional extrapolando o valor de Cs para idade de 27 anos. Na regressão dos valores medidos na idade de 27 anos foi desconsiderada a profundidade de 27,5 mm, pois o valor deste ponto apresenta uma distorção em relação ao restante do perfil.

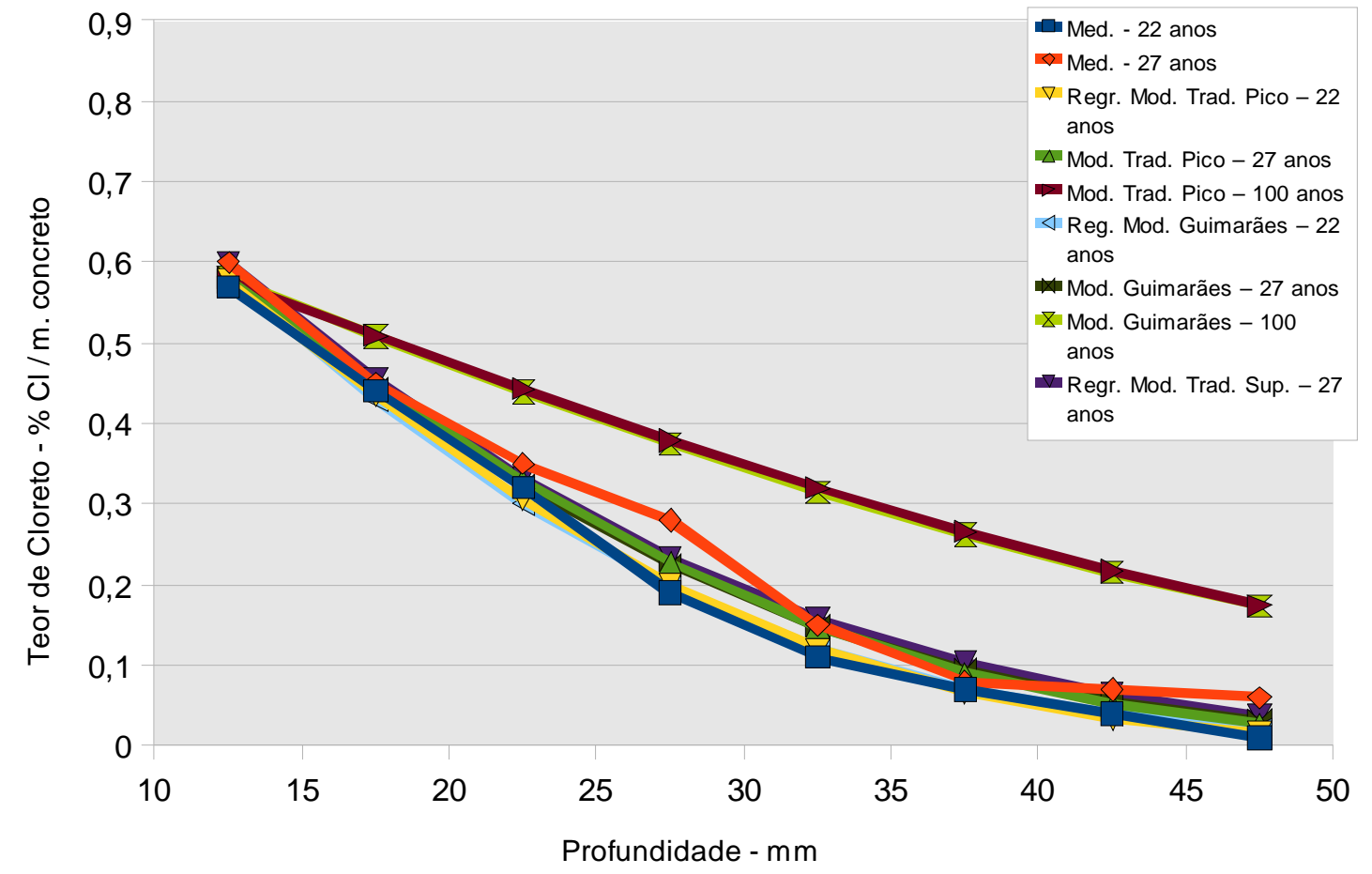

Figura 7: Perfis de cloretos medidos nas idades de 22 anos e 27 anos; curva de regressão obtida pelo modelo tradicional deslocando o eixo do x para o pico para idade de 22 anos; perfis estimados para 27 anos e 100 anos com modelo tradicional deslocando o eixo do x para o pico, conforme sugerido por ANDRADE et al. [1];perfis estimados para 27 anos e 100 anos utilizando modelo sugerido por GUIMARÃES e HELENE [4];

e curva de regressão obtida pelo modelo tradicional extrapolando o valor de Cs para idade de 27 anos, respectivamente às curvas citadas na legenda

\subsection{Análise dos resultados}

A Tabela 1 mostra que os valores do coeficiente de difusão do concreto (D) é consideravelmente diferente para os três modelos. Já o valor do teor de cloreto no pico são aproximadamente iguais para as curvas de regressão dos três modelos.

O modelo tradicional deslocando o eixo do x para pico, sugerido por ANDRADE et al. [1], o modelo tradicional extrapolando o valor de Cs, sugerido por NILSSON et al. [2] e o sugerido por GUIMARÃES e HELENE [4] apresentaram perfis de cloretos para as idades de 22 anos muito próximos, sendo que as curvas praticamente se sobrepoem com o perfil de cloretos medidos (Fig. 6 e Fig. 7). Na Fig. 7 observa-se que os dois modelos que deslocam o eixo do x para o pico, conforme sugere ANDRADE et al. [1] e proposto por GUIMARÃES e HELENE [4], apresentam também boa aproximação para perfis estimados para idade de 27 anos com a curva de regressão do perfil medido na mesma idade, com as três curvas praticamente se sobrepondo. O modelo tradicional extrapolando o valor de Cs, para idade de 27 anos, já apresenta um aumento no teor de cloretos para o teor no pico e todos os pontos de sua curva apresentam valores superiores aos outros dois modelos (Fig. 6 e Fig. 7). As curvas também apresentam valores muito próximos os perfis estimados para 100 anos para o modelo tradicional deslocando o eixo do x para o pico e para o modelo proposto por GUIMARÃES e HELENE [4] (Fig. 7). O modelo tradicional extrapolando o 
valor de Cs, além de apresentar elevado teor de cloretos no pico para idade de 100 anos, estima valores bem maiores de cloretos ao longo de todo perfil, quando comparado com os outros dois modelos (Fig. 6 e Fig. 7).

\section{CONCLUSÕES}

O modelo tradicional da solução da segunda lei de Fick, utilizando Cs extrapolado para a superfície da estrutura, sugerido por NILSSON et al. [2], conforme Figura 1, utilizando a eq. 1, não é adequado para estimar a vida útil de estruturas de concreto que apresentam perfil de cloreto formando pico, pois apresentam uma grande variação do teor no pico com o aumento da idade.

Com exceção do ponto na profundidade de $27,5 \mathrm{~mm}$, as curvas estimadas com o modelo tradicional situando o eixo do x no pico, sugerido por ANDRADE et al. [1]], e o modelo sugerido por GUIMARÃES e HELENE [4] apresentam boa aproximação com o perfil medido na idade de 27 anos e principalmente com sua curva de regressão.

Portanto estes dois modelos podem ser utilizados para estimar a vida útil residual, ou seja, para estimar a vida útil desde a inspeção de uma estrutura existente até a despassivação do aço, sendo mais fácil a utilização do modelo tradicional. Logo, no caso de avaliação de uma estrutura existente, sugere-se utilizar o modelo sugerido por ANDRADE et al. [1].

No entanto, quando o objetivo é obter o coeficiente de difusão do concreto (D), para estimar vida útil de projeto em concretos similares, ou quando estruturas são utilizadas em pesquisas, estes modelos devem ser analisados com cuidado. Observam-se valores de coeficientes de difusão diferentes entre os dois modelos para um mesmo perfil (Tabela 1) e, portanto, deve-se analisar qual modelo se adapta melhor a situação do perfil formando pico. Provavelmente o modelo sugerido por GUIMARÃES e HELENE [4] seja mais aplicável quando há forte dessanilização, ou seja, perda de cloreto para o meio antes do pico, como o estudo de caso apresentado neste trabalho. O modelo tradicional situando o eixo do x no pico, sugerido por ANDRADE et al. [1]], deve-se aplicar quando há formação de pico sem perdas de cloreto para o meio antes do pico, sendo o pico formado devido a fatores como carbonatação, má qualidade da camada mais externa do concreto, entre outros.

\section{BIBLIOGRAFIA}

[1] ANDRADE, C., SAGRERA, J.L., SANJUÁN, M.A., "Several years study on chloride ion penetration into concrete exposed to Atlantic Ocean water”, In: $2^{\text {nd }}$ International Rilem Workshop on Testing and Modelling the Chloride Ingress into Concrete, Eds. C.Andrade, J.Kropp, Paris, CD 2000.

[2] NILSSON, L., ANDERSEN, A., LUPING, T., UTGENANNT, P., "Chloride ingress data from field exposure in a swedish road environment”, In: $2^{\text {nd }}$ International Rilem Workshop on Testing and Modelling the Chloride Ingress into Concrete, Eds. C.Andrade, J.Kropp. Paris, CD 2000.

[3] GUIMARÃES, A.T.C., HELENE, P.R.L., Influência do teor de umidade sobre a difusividade de cloretos na pasta de cimento, Relatório FAPESP processo 99/10909-7, São Paulo, 2001.

[4] GUIMARÃES, A.T.C., HELENE, P.R.L., “Concrete structures service life estimation wich chloride peak profiles”, In: Quality of Concrete Structures and Recent Advances in Concrete Materials and Testing, ACI, Recife, September 2005.

[5] CRANK, J., The mathematics of diffusion, New York, Oxford University Press, 1975.

[6] BRETANHA, S., Variação do grau de saturação do concreto em ambiente marítimo. Dissertação de M.Sc., Fundação Universidade Federal do Rio Grande, Rio Grande, RS, Brasil, 2004.

[7] AMERICAN SOCIETY FOR TESTING OF MATERIALS. "Standart test method for obtaining and testing drilled cores and sawed beams of concrete - ASTM C 42-94”, In: Annual Book of ASTM Standard, Philadelphia, 1994.

[8] AMERICAN SOCIETY FOR TESTING OF MATERIALS. "Standard test method for specific gravity, absorption, and voids in hardened concrete - ASTM C 642-90”, In: Annual Book of ASTM Standard, Philadelphia, 1990. 
[9] GUIMARÃES, A.T.C., Vida útil de estruturas de concreto armado em ambientes marítimos, Tese de D.Sc., PCC/USP, São Paulo, SP, Brasil, 2000.

[10] AMERICAN SOCIETY FOR TESTING OF MATERIALS, "Standart test method for water-soluble chloride in mortar and concrete - ASTM C 1218-92”, In: Annual Book of ASTM Standard. Philadelphia, 1993. 